\title{
Optimal Coordination of Overcurrent Directional and Distance Relays in Meshed Networks Using Genetic Algorithm
}

\author{
M. H. Marcolino, J. B. Leite and J. R. S. Mantovani, Member, IEEE
}

\begin{abstract}
Protection of transmission lines is essential to ensure a correct operation of power systems. In this paper a pilot protection scheme, known as permissive overreaching transfer trip, is used. This structure combine two different protection relays: directional overcurrent relay, a backup protection for phase-earth faults, and distance relay for phase-phase faults. An intelligent method was developed to the optimal coordination of protection relays in meshed networks like transmission networks. The proposed method employs the genetic algorithm as optimization procedure that considers network characteristics (maximum load current and minimum fault current) and adjustment parameters of overcurrent and distance relays. Two testing networks, one of 8-bus and other of 39-bus, were used to evaluate the proposed method that obtained feasible and good solutions for the coordination problem.
\end{abstract}

Keywords - Power system protection, optimal coordination, overcurrent and distance relays, modeling of numerical relay, genetic algorithm.

\section{INTRODUÇÃO}

$\mathrm{N}^{2}$ a proteção de sistemas de extra alta tensão, os relés de distância (DR) devem atuar com o menor tempo de atraso possível durante a isolação de faltas devido aos efeitos operacionais e dinâmicos no sistema elétrico. Desta forma, o funcionamento apropriado de um sistema de proteção com essa característica é garantido usando o esquema de proteção piloto de transferência de trip de sobrealcance permissivo (POTT), Fig. 1, que utiliza relés de distância para proteção contra faltas entre fases e relés de sobrecorrente direcionais (DOCR) para proteção de retaguarda de faltas à terra [1].

Os DRs são amplamente utilizados em esquemas de proteção por serem eficientes e rápidos na isolação de faltas e economicamente vantajosos quando comparados com outros tipos de relés. Além disso, estes relés são facilmente ajustados para as funções de proteção principal e de retaguarda e, diferente dos relés de sobrecorrente, os DRs são afetados pela impedância de Thévenin [2]. Por outro lado, os DOCRs são intensamente empregados na função proteção de retaguarda em sistemas de transmissão devido os seus custos e à flexibilidade de ajuste dos seus parâmetros de controle na coordenação deste tipo de relés.

A coordenação dos relés primários e de retaguarda deve ser realizada de modo a garantir a operação adequada do sistema protegido. O intervalo de tempo de coordenação (CTI) na proteção de retaguarda de sobrecorrente assegura a seletividade do esquema de proteção impedindo a atuação da proteção de

Este trabalho foi financiado pela CAPES, FEPISA (Proc. 032/2012), FAPESP (Proc. 2013/23590-8) e CNPQ (Proc. 305371/2012-6).

M. H. Marcolino, Universidade Estadual Paulista - UNESP, Ilha Solteira, SP, Brasil, matheus.feis@gmail.com retaguarda antes da principal. Em redes malhadas, a identificação prévia de todos os pares de relés principal/retaguarda $(\mathrm{P} / \mathrm{R})$ é uma etapa importante para coordenar corretamente os relés. $\mathrm{Na}$ literatura, este procedimento é conhecido como break point set (BPS) sendo realizado através de diferentes métodos heurísticos que empregam a teoria dos grafos [3], [4].

A coordenação ótima dos relés de proteção pode ser formulada através de um modelo de otimização cujas propostas de solução encontradas na literatura são técnicas que utilizam a programação linear e meta-heurísticas como os algoritmos evolutivos (AE) [5], algoritmos genéticos (GA) [6], [7] e particle swarm optimization (PSO) [8]. Pesquisa recente propõe

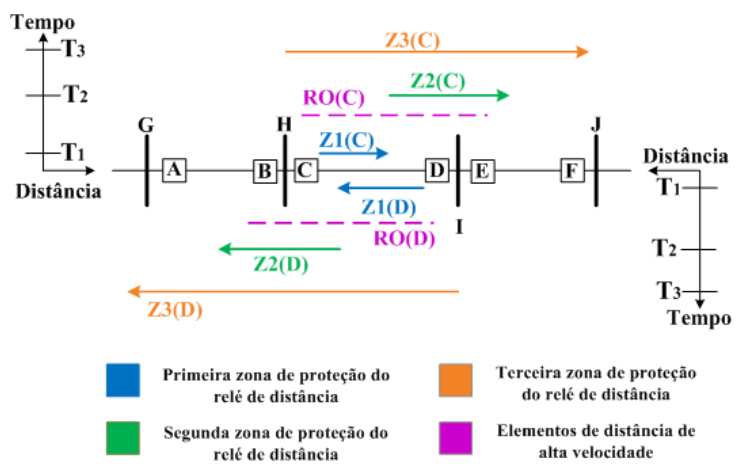

Figura 1. Alcance das zonas de distância no esquema POTT.

uso do nondominated sorting genetic algorithm-II (NSGAII) na solução do problema de coordenação de DOCRS. O NSGA-II utiliza o conceito de dominância e frente de Pareto e apresenta tempos de operação reduzidos quando comparado a outros métodos [9]. Encontra-se também na literatura uma técnica hibrida que utiliza algoritmo evolutivo populacional chamado de otimização biogeométrica com programação linear para coordenar DOCR em redes de maior complexidade, esta técnica inovadora apresenta resultados eficientes [10].

Neste trabalho, é proposto um modelo matemático modificado caracterizado pelo POTT (Fig. 1) para o problema de coordenação de DRs e DOCR em sistemas de transmissão de energia elétrica considerando-se neste modelo todos pares $\mathrm{P} / \mathrm{R}$ característicos da rede elétrica. Para solução do modelo proposto desenvolve-se um algoritmo genético dedicado.

Este trabalho além desta introdução é composto por cinco seções. Na seção II, o problema de coordenação ótima para sistemas de transmissão malhados é apresentado através de um

J. B. Leite, Universidade Estadual Paulista - UNESP, Ilha Solteira, SP, Brasil, jonatasboasleite@gmail.com

J. R. S. Mantovani, Universidade Estadual Paulista - UNESP, Ilha Solteira, SP, Brasil, ant@dee.feis.unesp.br 
exemplo, em seguida o problema é estruturado e formulado matematicamente. Na seção III detalha-se a técnica do GA empregada, descrevendo cada etapa do processo de otimização. As seções IV e V apresentam, respectivamente, os resultados e as conclusões da utilização desta estrutura de proteção para dois sistemas teste, um com 8 e outro com 39 barras.

\section{O PROBLEMA DE COORDENAÇÃO}

A coordenação de relés de proteção em sistemas malhados é complexa, e envolve um conjunto de regras e técnicas essenciais para garantir a eficiência da proteção. Os relés de sobrecorrentes utilizados pelo esquema POTT devem ser direcionais e cada par de DOCR deve ser coordenado. Nas barras onde há fontes externas interconectadas à malha, os relés pertencentes a esta interconexão devem ser coordenados com os relés localizados no interior da malha. A coordenação é efetuada através de uma movimentação partindo da montante em direção da jusante da malha, onde as características inerentes do sistema (localização e influência da impedância das fontes de geração, linhas de transmissão, transformadores) definem uma orientação.

\section{A. Construção do BPS}

Nesta seção, apresenta-se, com base na literatura [3] e [4], o problema da coordenação de relés em sistemas malhados. Por exemplo, na Fig. 2 é ilustrado um sistema básico que possui 4 barras e 5 linhas de transmissão. Os DOCRs e DRs instalados em cada extremidade das linhas $\left(R_{1}, \ldots, R_{10}\right)$ desempenham simultaneamente as funções de proteção principal e de retaguarda.

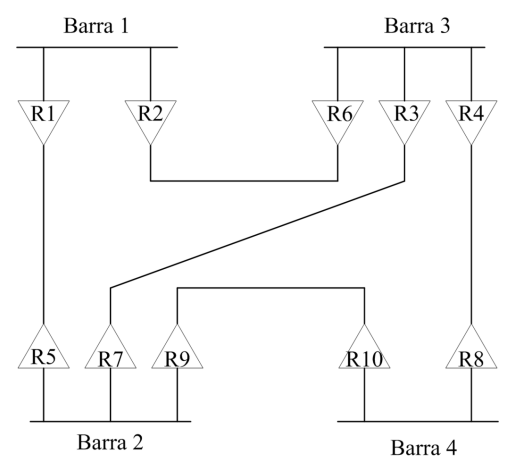

Figura 2. Exemplo de rede elétrica malhada [4].

Percorrendo as malhas do sistema básico, apresentado na Fig. 2, pode-se definir 16 pares $(\mathrm{P} / \mathrm{R})$ de relés desempenhando as funções de primário $(\mathrm{P})$ e retaguarda $(\mathrm{R})$. A coordenação dos DRs e DOCRs envolve obter todos os possíveis pares $(\mathrm{P} / \mathrm{R}) \mathrm{de}$ relés do sistema. Por exemplo, os 16 pares do sistema básico são denotados como (R7/R1), (R9/R1), (R3/R2), (R4/R2), (R5/R3), (R9/R3), (R10/R4), (R2/R5), (R1/R6), (R4/R7), (R6/R7), (R3/R8), (R6/R8), (R8/R9), (R5/R10), (R7/R10). Assim, a parametrização do relé 1 requer o ajuste do relé 6 , pois eles formam o par (R1/R6). Do mesmo modo, os parâmetros dos relés 7 e 8 estão vinculados aos ajustes do relé 6 . Estes relés também devem satisfazer os intervalos de coordenação dos pares (R4/R7) e (R3/R8). Para realizar os ajustes dos relés de forma correta, ou seja, respeitando as restrições inerentes ao problema da coordenação entre DRs e DOCRs, é necessária que a etapa BPS seja incluída no algoritmo e o problema seja formulado respeitando todas as condições operacionais e topológicas do sistema.

Para estruturar o problema, definem-se as matrizes $\boldsymbol{P}$ e $\boldsymbol{R}$ relacionadas, respectivamente, à proteção principal e de retaguarda. Estas matrizes são formadas de acordo com as quantidades de pares $(\mathrm{P} / \mathrm{R})$, número de linhas das matrizes, e de relés da rede, número de colunas das matrizes. Portanto, as matrizes $\boldsymbol{P}$ e $\boldsymbol{R}$ devem ter 16 linhas e 10 colunas para o sistema básico da Fig. 2.

$$
\begin{gathered}
\boldsymbol{P}=\left[p_{i j}\right]_{n p \times n r}=\left[\begin{array}{ccc}
p_{1 \times 1} & \cdots & p_{1 \times n r} \\
\vdots & \ddots & \vdots \\
p_{n p \times 1} & \cdots & p_{n p \times n r}
\end{array}\right] \\
\boldsymbol{R}=\left[r_{i j}\right]_{n p \times n r}=\left[\begin{array}{ccc}
r_{1 \times 1} & \cdots & r_{1 \times n r} \\
\vdots & \ddots & \vdots \\
r_{n p \times 1} & \cdots & r_{n p \times n r}
\end{array}\right]
\end{gathered}
$$

Estas matrizes são compostas por valores 1 (válido) e 0 (não válido), atribuídos de acordo com cada par para que seja possível identificar na formulação do problema qual o relé com função principal e o relé de retaguarda para cada par. Por exemplo, para o primeiro o par de relés (R7/R1), o elemento $p_{1 \times 7}$ é atribuído o valor 1 , aos outros elementos da linha $1 \mathrm{da}$ matriz $\boldsymbol{P}$ são atribuídos o valor 0 . O mesmo procedimento vale para a matriz $\boldsymbol{R}$, o mesmo par (R7/R1) indica que o elemento $r_{1 \times 1}$ é 1 e os outros elementos da linha 1 são zero, e assim sucessivamente, até a matriz ser formada por completo.

\section{B. Formulação do problema}

A formulação do problema depende diretamente das curvas características de proteção dos DRs e DOCRs e da relação entre essas curvas que determinam o tempo de abertura do relé em função da magnitude do fluxo de corrente. Na Fig. 3 apresentase um exemplo da relação entre estas curvas.

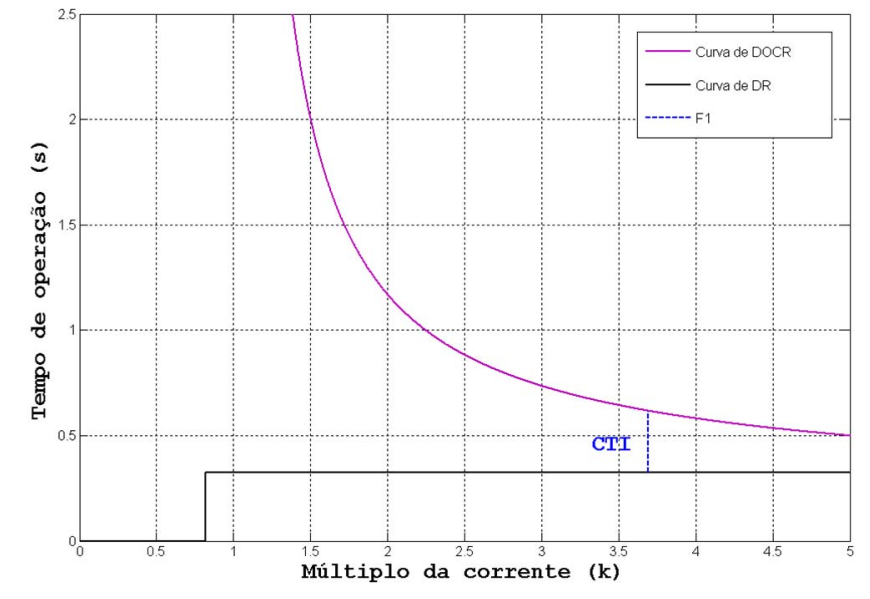

Figura 3. Curvas características usadas na coordenação dos relés.

O comportamento de cada relé é determinado pela condição operacional do sistema. Na Fig. 3 ilustra-se uma condição de falta (F1), onde a segunda zona do DR deve isolar a contingência para reparos, e se isto não ocorrer a proteção de retaguarda referente à curva de DOCR deve realizar esta tarefa após um intervalo de tempo de CTI segundos.

A relação entre as funções de proteção principal (DR) e de retaguarda (DOCR) é definida pelo atraso temporal entre a 
atuação da proteção principal e a atuação da retaguarda para uma mesma magnitude da corrente de curto-circuito, representada pelo múltiplo de corrente, $k$, que é a razão entre a corrente de curto-circuito e a corrente de ajuste do relé. Este atraso temporal, conhecido como CTI, é uma das principais restrições do problema de coordenação de relés.

A função objetivo (FO) da formulação matemática do problema de coordenação ótima de relés minimiza o tempo de operação dos relés de sobrecorrente, conforme a equação (3).

$$
\min F O=\alpha \sum_{j=1}^{n r} t_{O P, j}+\beta \sum_{i=1}^{n p}\left|\Delta t_{C C-D 2, i}-\right| \Delta t_{C C-D 2, i}||
$$

Sendo:

$$
\begin{gathered}
\Delta t_{C C-D 2, i}=\sum_{j=1}^{n r} r_{i j} t_{O P, j}-\sum_{j=1}^{n r} p_{i j} T_{Z 2, j}-C T I_{j}, \quad i=1,2, \ldots n p \\
t_{O P, j}=\frac{a T M S_{j}}{\left(\frac{I_{C C, j}}{I_{A, j}}\right)^{n}-1}, \quad j=1,2, \ldots n r
\end{gathered}
$$

$$
\left[\Delta t_{C C-D 2}\right]_{i x 1}=\left\{[R]_{i x j}\left[T_{O P}\right]_{j x 1}-[P]_{i x j}\left[T_{Z 2}\right]_{j x 1}\right\}-[C T I]_{i x 1}
$$

O primeiro termo da FO representa o tempo de operação dos relés de sobrecorrente, $t_{O P, j}$, onde $n r$ é o número de relés. Em (5), o tempo de operação é calculado usando o fator multiplicativo de ajuste de tempo, $T M S_{j}$, a relação $I_{C C, j} / I_{A, j}$ que representa o múltiplo da corrente, onde $I_{C C, j}$ é a corrente de curto-circuito do relé $j$ e $I_{A, j}$ é a corrente de ajuste do relé $j$, e os fatores $a$ e $n$ que caracterizam os diversos tipos de relés de sobrecorrente definidos pelo padrão IEC 60255 e cujos valores são apresentados na Tabela I [2].

TABELA I. VALORES DO IEC PARA RELÉS DE SOBRECORRENTES.

\begin{tabular}{cccc}
\hline \hline Tipo & Tipo $\left(\mathbf{n}^{\mathbf{0}}\right)$ & Fator $\boldsymbol{a}$ & Fator $\boldsymbol{n}$ \\
\hline Extremamente inverso & 1 & 80 & 2 \\
Muito inverso & 2 & 13.5 & 1 \\
Inverso padrão & 3 & 0.14 & 0.02 \\
Tempo definido & 4 & 120 & 1 \\
\hline \hline
\end{tabular}

Em geral, o relé inverso padrão responde com uma margem satisfatória para a maioria dos curtos-circuitos [1], porém os testes realizados demonstram que a intensidade da falta pode influenciar na sensibilidade dos relés que possuem os mesmos valores de $T M S$ e $I_{A}$. O tempo de operação do relé depende do múltiplo da corrente, ou seja, do valor da corrente que sensibiliza o relé e de sua corrente de ajuste, logo um relé responderá mais rápido que outro conforme a intensidade e o tipo da falta. Este critério é incluído no modelo matemático proposto para o problema de coordenação onde a escolha do tipo de relé é efetuada respeitando as restrições de coordenação no menor tempo possível.

$\mathrm{O}$ segundo termo da FO representa a restrição de coordenação entre o tempo da segunda zona do relé de distância, $T_{Z 2}$, e o tempo de operação do relé de retaguarda de sobrecorrente, $t_{O P}$, onde $n p$ é o número de pares $(\mathrm{P} / \mathrm{R})$ de relés no sistema. Se a diferença dos tempos de operação, $\Delta t_{C C-D 2, i}$, dada por (4), resultar um valor negativo, então o atraso temporal entre a atuação da proteção principal e a atuação da retaguarda deve ser inferior ao CTI violando, assim, a restrição de coordenação. Somente quando a diferença dos tempos de operação resultar num valor positivo, o segundo termo da FO será nulo impedindo sua penalização, com isso os pares $(\mathrm{P} / \mathrm{R})$ são coordenados adequadamente.

A equação (6) é a forma matricial compacta da diferença dos tempos de operação dada por (4). Esta representação matricial facilita a implementação computacional da técnica de solução proposta para resolver este problema de otimização.

Os parâmetros $\alpha$ e $\beta$ são fatores de penalidade utilizados para ponderar os dois termos da FO. O parâmetro $\alpha$ está relacionado com o tempo de operação dos relés de sobrecorrente. Se o valor de $\alpha$ é grande, maior é a probabilidade das soluções geradas terem pequeno tempo de operação. Por outro lado, o parâmetro $\beta$ relaciona-se com a coordenação entre a segunda zona do relé de distância principal e a função de sobrecorrente da retaguarda. Quanto maior o peso de $\beta$, maior será a precisão das soluções com relação a restrição de coordenação. O objetivo geral é procurar boas soluções que respeitem a restrição de coordenação com menor tempo possível de operação dos relés de sobrecorrente e de distância. Dessa forma, a parametrização dos relés também deve ser definida por restrições, como (7)(10).

$$
\begin{gathered}
T M S_{j}^{\text {min }} \leq T M S_{j} \leq T M S_{j}^{\text {max }}, \quad j=1,2, \ldots n r \\
I_{\text {carga }, j}^{\text {max }} \leq I_{A, j} \leq I_{\text {falta }, j}^{\text {min }}, \quad j=1,2, \ldots n r \\
1 \leq \text { iipo }_{j} \leq 4, \quad j=1,2, \ldots n r \\
T_{Z 2, j}^{\min } \leq T_{Z 2, j} \leq T_{Z 2, j}^{\max }, \quad j=1,2, \ldots n r
\end{gathered}
$$

Em (7), o fator multiplicativo de tempo, $T M S_{j}$, é representado por uma variável real de $T M S_{j}^{\min }=0,05$ a $T M S_{j}^{\max }=1 . \mathrm{Em}$ (8), a corrente $I_{A, j}$ também é uma variável real entre a corrente de carga máxima, $I_{c a r g a, j}^{\max }$, e o fluxo mínimo de corrente durante uma falta, $\mathrm{I}_{\text {falta,j }}^{\min }$. Na equação (9), a variável inteira Tipo $_{j}$ indica qual das curvas definidas pelo padrão IEC e apresentadas na Tabela I é utilizada pelo relé de sobrecorrente. Por exemplo, se Tipo $_{j}=1$, então o relé possui a característica de extremamente inverso onde $a=80$ e $n=2$. Estas restrições permitem o ajuste dos parâmetros dos DOCRs utilizados na FO.

O parâmetro $T_{Z 2, j}$ corresponde ao tempo de atuação da segunda zona dos DRs, pois na primeira zona a atuação dos DRs é considerada instantânea, $T_{Z 1, j}=0 s$. O tempo de atuação da segunda zona é representado usando uma variável real limitada por valores práticos no intervalo de $T_{Z 2, j}^{\min }=200 \mathrm{~ms}$ a $T_{Z 2, j}^{\max }=400 \mathrm{~ms}$ como dado por (10) [2].

Para solução deste problema de otimização matemática propõe-se um GA dedicado, que dentre todas as ferramentas de otimização disponíveis é uma das mais consolidadas para este tipo de problema. Este algoritmo é uma ferramenta robusta para resolver problemas não lineares e deve obter boas soluções com baixo tempo de processamento para o problema de coordenação de relés de proteção. 


\section{TÉCNICA DE SOLUÇÃO}

O GA utiliza uma população formada por um conjunto de indivíduos que, por sua vez, são constituídos por cromossomos resultantes da codificação das possíveis soluções do problema de otimização. As características específicas de cada indivíduo determinam sua capacidade de sobrevivência que é determinada pelo seu conteúdo genético, também conhecido na biologia como gene cujos agrupamentos formam os cromossomos. A população é capaz de evoluir devido à aplicação de operadores genéticos, como: seleção, recombinação e mutação, em cada indivíduo durante sua reprodução. O princípio da seleção natural permite gerar descendentes somente dos melhores indivíduos. No processo de recombinação genética há a duplicação e separação de células reprodutivas gerando uma metade de célula, e. $g$. espermatozóide, que na fecundação se junta com outra metade formando o zigoto. Nesse processo ocorre uma troca de material genético entre os parceiros levando à geração de um indivíduo mais evoluído. Na mutação, o material genético da prole pode sofrer uma modificação pontual e imprevisível. Os indivíduos mais fortes sobrevivem durante o ciclo geracional do GA, propiciando o aparecimento de indivíduos mais adaptados, ou seja, melhorando as soluções do problema de otimização [7].

Na metodologia proposta, os dados topológicos da rede são parâmetros de entrada para a realização da etapa BPS, após a determinação correta dos pares (P/R), o GA é inicializado. $\mathrm{Na}$ Fig. 4 ilustra-se a estrutura de codificação do cromossomo utilizado pelo GA bem como as variáveis (genes) que representam os parâmetros de configuração dos relés. Os genes de cada indivíduo são, respectivamente, a corrente de ajuste do relé de sobrecorrente, o fator multiplicativo de ajuste de tempo, o tipo do relé de sobrecorrente e o tempo de atuação da segunda zona.

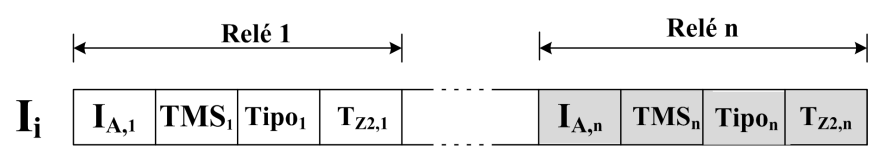

Figura 4. Estrutura de codificação do cromossomo.

O GA proposto consiste de 6 etapas: inicialização, cálculo da função objetivo, seleção, recombinação, mutação e validação do(s) critério(s) de parada, que são apresentadas a seguir:

1) Inicialização. Gerar a população inicial de forma aleatoriamente controlada, atendendo-se as restrições de (7) a (10);

2) Cálculo da função objetivo. A função objetivo é calculada para cada indivíduo utilizando-se as correntes de curtocircuito para a obtenção dos tempos dos dispositivos de proteção. Essas correntes são previamente obtidas através de simulações de faltas no sistema usando o software ATP;

3) Seleção por torneios. Realizam-se $n$ torneios ( $n$ - tamanho da população) onde em cada um são sorteados quatro indivíduos e aquele que possuir menor valor da FO é selecionado. O mecanismo de torneio foi escolhido pela vantagem do pequeno esforço computacional e pelo fato de encontrar um número inteiro de descendentes;

4) Recombinação. O processo de recombinação utilizado é a recombinação de um único ponto, ou seja, a parcela de uma configuração é trocada com a parcela correspondente da outra configuração do mesmo par selecionado de acordo com a taxa de recombinação preestabelecida;

5) Mutação. Processo de mutação do tipo indutivo em que se efetua uma alteração no cromossomo de acordo com a taxa de mutação preestabelecida. Para efetuar a mutação, para cada um dos setores de variáveis que compõem o cromossomo, procede-se da seguinte maneira: cada indivíduo sofre uma pequena perturbação que pode ser realizada trocando a curva do relé, alterando o valor da corrente de ajuste do relé, ou o valor do tempo de atuação da segunda zona do relé de distância;

6) Critério de parada. Controlar a solução incumbente, se esta não apresenta melhoria durante um número preestabelecido de gerações o processo iterativo é considerado convergido. Outro critério utilizado pelo GA é o número máximo de gerações, ou seja, se o ciclo geracional do GA atinge um número preestabelecido de gerações, então a execução do algoritmo é terminada.

\section{RESUltados}

Os testes foram realizados usando um computador com processador Intel ${ }^{\circledR}$ Core $^{\mathrm{TM}}$ i7 $2.3 \mathrm{GHz}$ e $16 \mathrm{~GB}$ de memória RAM. Os sistemas de 8 barras e de 39 barras foram utilizados nos testes. O sistema de 8 barras possui 2 geradores de 150MVA, 7 linhas de transmissão, e 28 relés (14 de DR e 14 de DOCR) posicionados conforme ilustrado na Fig. 5.

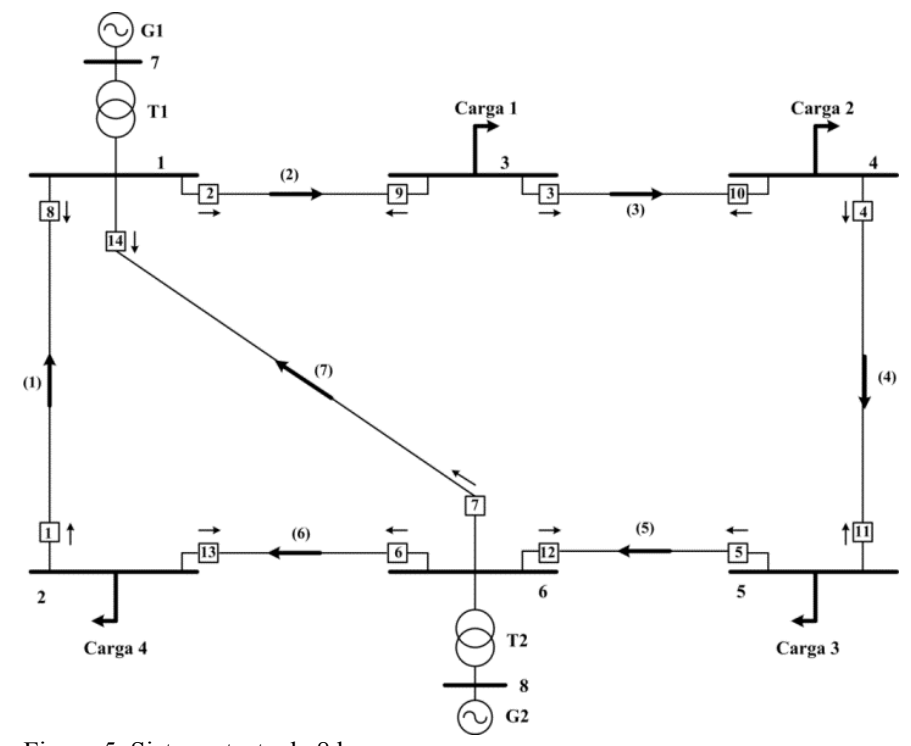

Figura 5. Sistema teste de 8 barras.

Os dados do sistema, como potência ativa e reativa das cargas, resistência e reatância das linhas de transmissão, dos transformadores e dos geradores e os pares (P/R) são fornecidos em [3], [8].

O sistema de 39 barras possui 34 linhas de transmissão, 10 geradores e 12 transformadores, conforme ilustrado na Fig. 6, todos os dados do sistema são fornecidos em [11].

$\mathrm{O}$ software $\mathrm{ATP}^{\circledR}$ foi utilizado para simular estes sistemas e calcular suas condições de carga e curto-circuito. O GA foi implementado utilizando o software $\mathrm{MatLab}^{\circledR}$. Os resultados das simulações realizadas no $\mathrm{ATP}^{\circledR}$ são utilizados pelo GA como parâmetros de entrada. De acordo com [2], o valor típico do CTI é igual á 0,3 segundos para relés numéricos. Os valores 


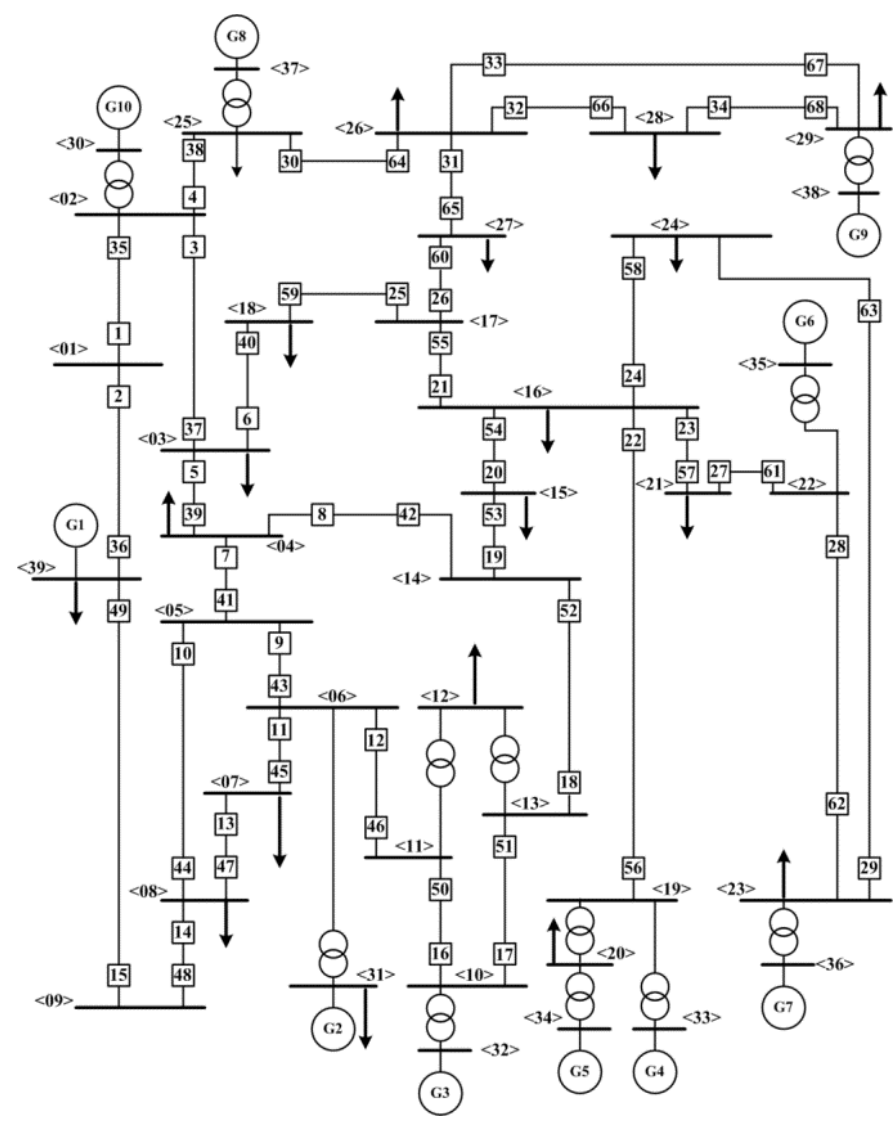

Figura 6. Sistema teste de 39 barras.

dos parâmetros $\alpha$ e $\beta$ da FO foram calibrados através de diversos testes, no final, obteve-se o melhor ajuste $(\alpha=1$ e $\beta=10)$ que corresponde aos valores finais usados neste trabalho para otimizar a coordenação de relés. Outros parâmetros de calibração do GA são apresentados na Tabela II.

TABELA II. PARÂMETROS DO GA.

\begin{tabular}{cc}
\hline \hline Parâmetros do GA & Valor \\
\hline Tamanho da população & 45 \\
Número de gerações & 100 \\
Taxa de Recombinação & $1 / 10$ \\
Taxa de Mutação & $3 \%$ \\
\hline
\end{tabular}

Os resultados apresentados nas Tabelas III e IV correspondem, respectivamente, aos parâmetros de configuração dos relés de sobrecorrentes e distância e aos intervalos de coordenação desses dispositivos, obtidos pelo GA para o sistema de 8 barras. Do mesmo modo, as Tabelas V e VI apresentam a resposta do GA para a coordenação otimizada do sistema de 39 barras.

Da segunda, terceira e quarta colunas da Tabela III pode-se concluir:

1) Os valores referentes às correntes de ajuste respeitam os limites impostos pela restrição (8), representando corretamente as características de carga da rede;

2) Todos os valores de $T M S$ são pequenos, o que indica uma atuação rápida dos relés sempre respeitando a faixa de ajuste, entre 0,05 e 1 ;
3) O tipo de relé DOCR mais utilizado, ou seja, aquele que apresentou melhor resposta temporal para este cenário foi o tipo 2 correspondente ao tipo muito inverso. $\mathrm{O}$ resultado obtido reflete a dependência das características do relé com o comportamento do sistema onde ele está localizado. Portanto, a inclusão de relés de sobrecorrentes com diferentes tipos de curvas características, de acordo com a Tabela I, viabiliza a avaliação de outras possibilidades de coordenação.

A última coluna apresenta os valores referentes ao relé de distância, ou seja, o tempo de atuação da segunda zona, pois a primeira zona foi considerada instantânea. Os resultados respeitam a restrição (10) e estão próximos do limite inferior de $T_{Z 2}^{\min }=200 \mathrm{~ms}$ onde são obtidas as soluções mais eficientes que possuem alta velocidade de atuação da proteção principal.

Os resultados das diferenças dos tempos de operação apresentados na Tabela IV são todos positivos e próximos à zero. O maior valor da diferença temporal é de 0,2589 , o que indica a correta obtenção dos pares $(\mathrm{P} / \mathrm{R})$ e a ausência de violação da restrição de coordenação em toda rede. Os valores mais próximos de zero representam maior precisão na isolação das faltas.

TABELA III. PARÂMETROS DOS RELÉS GERADOS PELO GA PARA O SISTEMA DE 8 BARRAS.

\begin{tabular}{|c|c|c|c|c|c|}
\hline $\begin{array}{l}N^{\circ} \\
\text { do } \\
\text { relé }\end{array}$ & $I_{A}(\mathrm{~A})$ & $\begin{array}{c}T M S \\
(\mathbf{s})\end{array}$ & Tipo & $T_{o p}(\mathbf{s})$ & $T_{Z 2}(\mathbf{s})$ \\
\hline 1 & 161,8126 & 0,2586 & 2 & 0,5446 & 0,2162 \\
\hline 2 & 370,9343 & 0,2050 & 2 & 0,5442 & 0,2366 \\
\hline 3 & 118,7370 & 0,1619 & 3 & 0,5191 & 0,2323 \\
\hline 4 & 182,8390 & 0,1193 & 2 & 0,5407 & 0,2161 \\
\hline 5 & 200,6075 & 0,0512 & 3 & 0,8120 & 0,2297 \\
\hline 6 & 541,2894 & 0,1463 & 1 & 0,5190 & 0,2533 \\
\hline 7 & 204,1378 & 0,2023 & 1 & 0,5456 & 0,2531 \\
\hline 8 & 274,1337 & 0,1327 & 2 & 0,5420 & 0,2347 \\
\hline 9 & 463,8704 & 0,0584 & 3 & 0,5419 & 0,2267 \\
\hline 10 & 180,8991 & 0,4322 & 2 & 0,5279 & 0,2469 \\
\hline 11 & 779,6485 & 0,0738 & 1 & 0,5526 & 0,2193 \\
\hline 12 & 467,3664 & 0,2546 & 2 & 0,5286 & 0,2412 \\
\hline 13 & 280,0940 & 0,1199 & 3 & 0,5596 & 0,2311 \\
\hline 14 & 491,9340 & 0,1284 & 2 & 0,5577 & 0,2400 \\
\hline \multicolumn{4}{|c|}{$\begin{array}{c}\text { Valor do Fitness } \\
7,836 \mathrm{~s}\end{array}$} & \multicolumn{2}{|c|}{$\begin{array}{c}\text { Média de } \mathrm{T}_{\mathrm{Z} 2} \\
0,234\end{array}$} \\
\hline
\end{tabular}

Os parâmetros de calibração do GA apresentados na Tabela II e usados para a coordenação otimizada do sistema de 8 barras também foram usados para o sistema de 39 barras. Os resultados da Tabela $\mathrm{V}$ revelam a robustez do algoritmo proposto na coordenação de um sistema grande. As correntes de ajuste respeitaram a restrição (8). Os valores de $T M S$ encontrados pelo GA foram majoritariamente inferiores a 500 ms, indicando a obtenção de soluções de boa qualidade com resposta temporal reduzida. Em comparação ao sistema de 8 barras onde houve maior ocorrência do relé de sobrecorrente do tipo 2, na coordenação do sistema de 39 barras o relé mais utilizado foi o tipo 3, que é o relé do tipo inverso padrão. Este resultado comprova a dependência das características do relé 
com o comportamento do sistema onde ele está localizado, como foi observado anteriormente.

Os valores referentes ao relé de distância encontrados pelo algoritmo respeitam a restrição (10) e estão muito próximos do limite inferior, $T_{Z 2}^{\min }=200 \mathrm{~ms}$, indicando a obtenção das melhores soluções possíveis, que favorecem uma resposta mais eficaz da proteção principal.

TABELA V. PARÂMETROS DOS RELÉS GERADOS PELO GA PARA O SISTEMA

\begin{tabular}{|c|c|c|c|c|c|}
\hline $\begin{array}{l}\mathbf{N}^{\circ} \text { do } \\
\text { relé }\end{array}$ & $I_{A}(\mathrm{~A})$ & $T M S$ (s) & Tipo & $T_{o p}(\mathrm{~s})$ & $T_{Z 2}(\mathrm{~s})$ \\
\hline 1 & 827,836 & 0,6681 & 1 & 0,6017 & 0,2603 \\
\hline 2 & 663,721 & 0,3818 & 2 & 0,5845 & 0,2539 \\
\hline 3 & 691,373 & 0,2088 & 3 & 0,5560 & 0,2477 \\
\hline 4 & 243,628 & 0,3224 & 3 & 0,5722 & 0,2569 \\
\hline 5 & 495,867 & 0,2476 & 3 & 0,5694 & 0,2459 \\
\hline 6 & 238,782 & 0,3149 & 3 & 0,5843 & 0,2241 \\
\hline 7 & 238,770 & 0,3073 & 3 & 0,6063 & 0,2445 \\
\hline 8 & 232,653 & 0,2714 & 3 & 0,5758 & 0,2485 \\
\hline 9 & 133,125 & 0,2667 & 4 & 0,6209 & 0,2581 \\
\hline 10 & 195,400 & 0,3158 & 3 & 0,5758 & 0,2507 \\
\hline 11 & 208,988 & 0,2988 & 3 & 0,5487 & 0,2581 \\
\hline 12 & 382,577 & 0,2463 & 3 & 0,5621 & 0,2531 \\
\hline 13 & 306,167 & 0,2344 & 3 & 0,5942 & 0,2416 \\
\hline 14 & 1531,864 & 0,0936 & 2 & 0,5370 & 0,2491 \\
\hline 15 & 1388,081 & 0,0667 & 3 & 0,5768 & 0,2167 \\
\hline 16 & 290,982 & 0,2418 & 3 & 0,5698 & 0,2588 \\
\hline 17 & 243,3531 & 0,2949 & 3 & 0,5994 & 0,2513 \\
\hline 18 & 299,402 & 0,2365 & 3 & 0,5729 & 0,2464 \\
\hline 19 & 181,030 & 0,3156 & 3 & 0,5885 & 0,2473 \\
\hline 20 & 518,918 & 0,2886 & 2 & 0,5848 & 0,2776 \\
\hline 21 & 284,710 & 0,2855 & 3 & 0,5656 & 0,2641 \\
\hline 22 & 1139,060 & 0,1224 & 1 & 0,1530 & 0,2696 \\
\hline $\begin{array}{l}\mathbf{N}^{0} \text { do } \\
\text { relé }\end{array}$ & $I_{A}(\mathrm{~A})$ & $T M S$ (s) & Tipo & $T_{o p}(\mathrm{~s})$ & $T_{Z 2}(\mathrm{~s})$ \\
\hline 23 & 282,4507 & 0,3081 & 3 & 0,5737 & 0,2735 \\
\hline 24 & 602,1326 & 0,8281 & 2 & 0,5852 & 0,2647 \\
\hline 25 & 132,434 & 0,3457 & 4 & 0,5704 & 0,2537 \\
\hline 26 & 220,728 & 0,3259 & 3 & 0,5843 & 0,2582 \\
\hline 27 & 280,721 & 0,2593 & 3 & 0,5695 & 0,2639 \\
\hline 28 & 116,300 & 0,3471 & 3 & 0,5919 & 0,2432 \\
\hline 29 & 37,524 & 0,4048 & 3 & 0,5783 & 0,2648 \\
\hline 30 & 321,856 & 0,2606 & 3 & 0,5709 & 0,2529 \\
\hline 31 & 370,585 & 0,2174 & 3 & 0,5578 & 0,2688 \\
\hline 32 & 19,300 & 0,5202 & 3 & 0,6166 & 0,2574 \\
\hline 33 & 51,797 & 0,3931 & 4 & 0,5900 & 0,2632 \\
\hline 34 & 167,023 & 0,2229 & 3 & 0,5744 & 0,2761 \\
\hline 35 & 640,007 & 0,4112 & 2 & 0,5774 & 0,2474 \\
\hline 36 & 761,735 & 0,2775 & 3 & 0,5654 & 0,2687 \\
\hline 37 & 802,548 & 0,3338 & 2 & 0,5687 & 0,2372 \\
\hline 38 & 266,590 & 0,5898 & 2 & 0,5466 & 0,2557 \\
\hline 39 & 551,828 & 0,1945 & 3 & 0,5432 & 0,2505 \\
\hline 40 & 230,971 & 0,2611 & 3 & 0,5593 & 0,2576 \\
\hline 41 & 272,536 & 0,0871 & 4 & 0,5588 & 0,2532 \\
\hline 42 & 227,535 & 0,1288 & 4 & 0,5933 & 0,2499 \\
\hline 43 & 94,767 & 0,3359 & 3 & 0,5664 & 0,2549 \\
\hline 44 & 214,624 & 0,3079 & 3 & 0,6041 & 0,2545 \\
\hline 45 & 220,335 & 0,1331 & 4 & 0,5767 & 0,2399 \\
\hline 46 & 341,355 & 0,2272 & 3 & 0,5627 & 0,2497 \\
\hline
\end{tabular}

\begin{tabular}{|c|c|c|c|c|c|}
\hline 47 & 324,272 & 0,1719 & 3 & 0,5487 & 0,2547 \\
\hline 48 & 1211,507 & 0,1084 & 3 & 0,5925 & 0,2715 \\
\hline 49 & 1010,749 & 0,6464 & 1 & 0,5878 & 0,2588 \\
\hline 50 & 348,573 & 0,7389 & 2 & 0,5813 & 0,2556 \\
\hline 51 & 273,657 & 0,2800 & 3 & 0,5607 & 0,2866 \\
\hline 52 & 347,676 & 0,9223 & 2 & 0,5868 & 0,2557 \\
\hline 53 & 183,494 & 0,2925 & 3 & 0,5695 & 0,2481 \\
\hline 54 & 595,965 & 0,2374 & 3 & 0,5665 & 0,2736 \\
\hline 55 & 261,227 & 0,2820 & 3 & 0,5828 & 0,2643 \\
\hline 56 & 1113,922 & 0,0619 & 3 & 0,5913 & 0,2817 \\
\hline 57 & 290,408 & 0,6813 & 1 & 0,5799 & 0,2388 \\
\hline 58 & 582,664 & 0,0680 & 3 & 0,6382 & 0,2291 \\
\hline 59 & 184,784 & 0,1458 & 4 & 0,5769 & 0,2728 \\
\hline 60 & 214,634 & 0,6329 & 2 & 0,5740 & 0,2414 \\
\hline 61 & 296,830 & 0,2297 & 3 & 0,5507 & 0,2745 \\
\hline 62 & 138,560 & 0,1595 & 4 & 0,5892 & 0,2545 \\
\hline 63 & 32,492 & 0,8135 & 4 & 0,5767 & 0,2722 \\
\hline 64 & 336,313 & 0,5191 & 2 & 0,5593 & 0,2677 \\
\hline 65 & 357,282 & 0,2345 & 3 & 0,5681 & 0,2580 \\
\hline 66 & 18,573 & 0,3620 & 3 & 0,5932 & 0,2345 \\
\hline 67 & 41,271 & 0,3304 & 3 & 0,5772 & 0,2725 \\
\hline 68 & 169,512 & 0,8178 & 2 & 0,5395 & 0,2740 \\
\hline
\end{tabular}

Os resultados das diferenças dos tempos de operação apresentados na Tabela VI são todos próximos de zero, indicando alta precisão do algoritmo utilizado. Mesmo quando a diferença temporal foi negativa e a restrição foi violada, os resultados não afetaram a coordenação dos relés, pois os valores obtidos sempre estiveram muito próximos de zero.

TABELA VI. LISTA DOS PARES (P/R) COM SEUS INTERVALOS DE COORDENAÇÃO PARA O SISTEMA DE 39 BARRAS.

\begin{tabular}{cccccccc}
\hline $\begin{array}{c}\mathbf{N}^{\mathbf{0}} \mathbf{d o} \\
\mathbf{p a r}\end{array}$ & $\mathbf{P}$ & $\mathbf{R}$ & $\begin{array}{c}\Delta \boldsymbol{t}_{\boldsymbol{C C}-\boldsymbol{D} \mathbf{2}} \\
\mathbf{( s )}\end{array}$ & $\begin{array}{c}\mathbf{N}^{\mathbf{0}} \mathbf{d o} \\
\mathbf{p a r}\end{array}$ & $\mathbf{P}$ & $\mathbf{R}$ & $\begin{array}{c}\Delta \boldsymbol{t}_{\boldsymbol{C C}-\boldsymbol{D} \mathbf{2}} \\
\mathbf{( s )}\end{array}$ \\
\hline 1 & 3 & 1 & 0,0539 & 25 & 18 & 17 & 0,0529 \\
2 & 4 & 1 & 0,0447 & 26 & 19 & 18 & 0,0255 \\
3 & 49 & 2 & 0,0256 & 27 & 42 & 18 & 0,0229 \\
4 & 5 & 3 & 0,0101 & 28 & 20 & 19 & 0,0108 \\
5 & 6 & 3 & 0,0318 & 29 & 21 & 20 & 0,0206 \\
6 & 30 & 4 & 0,0193 & 30 & 22 & 20 & 0,0151 \\
7 & 7 & 5 & 0,0248 & 31 & 23 & 20 & 0,0117 \\
8 & 8 & 5 & 0,0209 & 32 & 24 & 20 & 0,0208 \\
9 & 59 & 6 & 0,0115 & 33 & 25 & 21 & 0,0118 \\
10 & 9 & 7 & 0,0482 & 34 & 26 & 21 & 0,0074 \\
11 & 10 & 7 & 0,0563 & 35 & 27 & 23 & 0,0097 \\
12 & 19 & 8 & 0,0284 & 36 & 63 & 24 & 0,0129 \\
13 & 52 & 8 & 0,0207 & 37 & 40 & 25 & 0,0128 \\
14 & 11 & 9 & 0,0619 & 38 & 65 & 26 & 0,0263 \\
15 & 12 & 9 & 0,0669 & 39 & 28 & 27 & 0,0262 \\
16 & 14 & 10 & 0,0259 & 40 & 29 & 28 & 0,0271 \\
17 & 47 & 10 & 0,0203 & 41 & 58 & 29 & 0,0491 \\
18 & 13 & 11 & 0,0071 & 42 & 31 & 30 & 0,0021 \\
19 & 50 & 12 & 0,0065 & 43 & 32 & 30 & 0,0135 \\
20 & 14 & 13 & 0,0451 & 44 & 33 & 30 & 0,0077 \\
21 & 44 & 13 & 0,0397 & 45 & 60 & 31 & 0,0164 \\
22 & 15 & 14 & 0,0206 & 46 & 34 & 32 & 0,0404 \\
23 & 36 & 15 & 0,0081 & 47 & 68 & 33 & 0,0159 \\
24 & 46 & 16 & 0,0201 & 48 & 67 & 34 & 0,0018 \\
49 & 2 & 35 & 0,0235 & 82 & 23 & 55 & 0,0097 \\
& & & & & & &
\end{tabular}




\begin{tabular}{|c|c|c|c|c|c|c|c|}
\hline 50 & 1 & 36 & 0,0051 & 83 & 24 & 55 & 0,0181 \\
\hline 51 & 4 & 37 & 0,0118 & 84 & 54 & 55 & 0,0092 \\
\hline 52 & 35 & 37 & 0,0213 & 85 & 21 & 56 & 0,0271 \\
\hline 53 & 3 & 38 & $-0,001$ & 86 & 23 & 56 & 0,0182 \\
\hline 54 & 35 & 38 & $-0,007$ & 87 & 24 & 56 & 0,0266 \\
\hline 55 & 6 & 39 & 0,019 & 88 & 54 & 56 & 0,0177 \\
\hline 56 & 37 & 39 & 0,0059 & 89 & 21 & 57 & 0,0158 \\
\hline 57 & 5 & 40 & 0,0134 & 90 & 22 & 57 & 0,0103 \\
\hline 58 & 37 & 40 & 0,0221 & 91 & 24 & 57 & 0,0152 \\
\hline 59 & 8 & 41 & 0,0103 & 92 & 54 & 57 & 0,0063 \\
\hline 60 & 39 & 41 & 0,0082 & 93 & 21 & 58 & 0,0740 \\
\hline 61 & 7 & 42 & 0,0487 & 94 & 22 & 58 & 0,0686 \\
\hline 62 & 39 & 42 & 0,0427 & 95 & 23 & 58 & 0,0652 \\
\hline 63 & 10 & 43 & 0,0163 & 96 & 54 & 58 & 0,0646 \\
\hline 64 & 41 & 43 & 0,0131 & 97 & 26 & 59 & 0,0186 \\
\hline 65 & 9 & 44 & 0,0459 & 98 & 55 & 59 & 0,0125 \\
\hline 66 & 41 & 44 & 0,0508 & 99 & 25 & 60 & 0,0202 \\
\hline 67 & 12 & 45 & 0,0236 & 100 & 55 & 60 & 0,0096 \\
\hline 68 & 43 & 45 & 0,0218 & 101 & 57 & 61 & 0,0118 \\
\hline 69 & 11 & 46 & 0,0045 & 102 & 61 & 62 & 0,0146 \\
\hline 70 & 43 & 46 & 0,0077 & 103 & 62 & 63 & 0,0221 \\
\hline 71 & 45 & 47 & 0,0087 & 104 & 38 & 64 & 0,0035 \\
\hline 72 & 44 & 48 & 0,0379 & 105 & 32 & 65 & 0,0105 \\
\hline 73 & 47 & 48 & 0,0377 & 106 & 33 & 65 & 0,0047 \\
\hline 74 & 48 & 49 & 0,0163 & 107 & 64 & 65 & 0,0002 \\
\hline 75 & 17 & 50 & 0,029 & 108 & 31 & 66 & 0,0243 \\
\hline 76 & 16 & 51 & 0,0019 & 109 & 33 & 66 & 0,0300 \\
\hline 77 & 51 & 52 & $-0,005$ & 110 & 64 & 66 & 0,0255 \\
\hline 78 & 42 & 53 & 0,0195 & 111 & 31 & 67 & 0,0084 \\
\hline $\begin{array}{c}\mathbf{N}^{0} \text { do } \\
\text { par }\end{array}$ & $\mathbf{P}$ & $\mathbf{R}$ & $\begin{array}{c}\Delta t_{C C-D 2} \\
\text { (s) }\end{array}$ & $\begin{array}{c}N^{\circ} \text { do } \\
\text { par }\end{array}$ & $\mathbf{P}$ & $\mathbf{R}$ & $\begin{array}{c}\Delta t_{C C-D 2} \\
\text { (s) }\end{array}$ \\
\hline 79 & 53 & 53 & 0,0138 & 112 & 32 & 67 & 0,0198 \\
\hline 80 & 53 & 54 & 0,0184 & 113 & 64 & 67 & 0,0095 \\
\hline 81 & 22 & 55 & 0,0131 & 114 & 66 & 68 & 0,0056 \\
\hline
\end{tabular}

As técnicas de otimização utilizadas com frequência na literatura para o problema de ajuste de relés são programação linear (PL) e PSO [8]. Análise quantitativa foi realizada para o sistema de 8 barras da Fig. 6, onde foi comparado o método proposto neste trabalho com os métodos PL e PSO híbrido, propostos em [8].

Os valores referentes a cada $\mathrm{DR}$, ou seja, $\mathrm{T}_{\mathrm{Z} 2}$ são comparados para cada método, Fig. 7 onde pode-se visualizar o resultado. $\mathrm{O}$ valor médio do método 1 , proposta do trabalho é 0,23 segundos. Para o método 2, que utiliza PL a média é 0,7 segundos e para o método 3 que utiliza PSO híbrido a média é 0,65 segundos. Verifica-se que ocorre uma melhoria significativa na velocidade de atuação do DR para o modelo proposto neste trabalho.

$\mathrm{O}$ valor do fitness é um fator importante na análise do desempenho da proteção. Este valor engloba as funções DR e DOCR, ou seja, o sistema de proteção. No método proposto o valor de fitness obtido foi de 7,836 que é aproximadamente 1,8 vezes mais rápido que o método $\mathrm{PSO}$ híbrido $(\mathrm{FO}=14,2)$ e 2 vezes mais rápido que $\mathrm{PL}(\mathrm{FO}=15,738)$.

\section{CONCLUSÕES}

No algoritmo desenvolvido utilizam-se as características principais do esquema de proteção POTT além de considerar

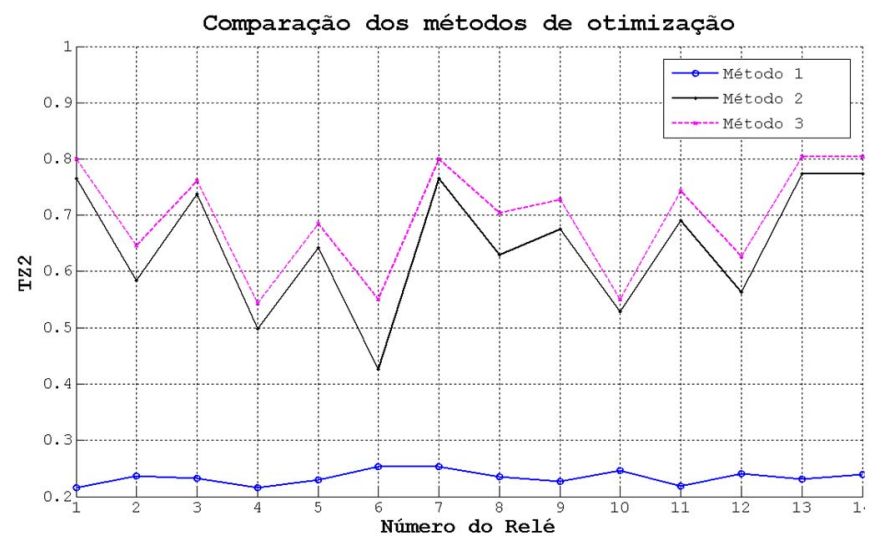

Figura 7. Comparação de $\mathrm{T}_{\mathrm{Z2}}$.

uma nova formulação da função objetivo, caracterizando de forma adequada e real o problema de coordenação da proteção de distância e sobrecorrente em redes malhadas. O GA padrão foi utilizado para resolver o modelo de otimização proposto considerando a inclusão das diversas características do sistema no algoritmo, além de diferentes funções de sobrecorrentes.

Os resultados apresentados revelam a eficiência do método proposto em comparação com outros métodos encontrados na literatura, vide Fig. 8. Sua eficiência se deve às modificações da função objetivo que mesmo usando muitas variáveis de decisão permitiu ao GA encontrar soluções de ótima qualidade com um número reduzido de iterações. Ao considerar as características das cargas na rede e os diversos tipos de relés assim como uma função objetivo que representa o problema de coordenação em redes malhadas de forma real, o GA padrão encontrou soluções de boa qualidade para os dois sistemas testes estudados, indicando a importância de uma formulação adequada para o problema de coordenação e a utilização de mais variáveis envolvendo os DOCRs.

\section{REFERÊNCIAS}

[1] P. M. Anderson, Power System Protection. New York: McGraw-Hill, 1999.

[2] AREVA, Network Protection \& Automation Guide, First edition, July 2002.

[3] A. S. Braga, and J. T. Saraiva. Coordination of Overcurrent Diretional Relays in Meshed Networks Using the Simplex Method. IEEE Eletrotechnical Conference, 1996, Vol. 3.

[4] K. Kawahara, H. Sasaki, and H. Sugihara. An Application of Rule Based System to the Coordination Of Directional Overcurrent Relays. IEEE Power System Protection Conference, 1997, No. 434.

[5] C. W. So and K. K. Li, "Time coordination method for power system protection by evolutionary algorithm", IEEE Trans. In. Appl., vol. 36, no. 5, pp. 1235-1240, Sep. 2000.

[6] R. M. Chabanloo, H. A. Abyaneh, S. S. H. Kamangar. A New Genetic Algorithm Method for Optimal Coordination of Overcurrent and Distance Relays Considering Various Characteristics for Overcurrent Relays. IEEE International Conference om Power and Energy, 2008.

[7] A. S. Noghabi, J. Sadeh, and H. R. Mashhadi. Considering Different Network Topologies in Optimal Overcurrent Relay Coordination Using a Hybrid GA. IEEE Transactions on Power Delivery, 2009, Vol. 24.

[8] J. Sadeh, V. Amintojjar, and M. Bashir. Coordination of Overcurrent and Distance Relays Using Hybrid Particle Swarm Optimization. IEEE International Conference on Advanced Power System Automation and Protection, 2011.

[9] Z. Moravej, F. Adelnia and F. Abbasi. Optimal coordination of directional overcurrent relays using NSGA-II. Electric Power Systems Research, Vol. 119, pp. 228-236, 2015.

[10] F. A. Albasri, A. R. Alroomi and J. H. Talaq. Optimal Coordination of Directional Overcurrent Relays Using Biogeography-Based Optimization 
Algorithms. IEEE Transactions on Power Delivery, vol. 30, no. 4, Aug. 2015.

[11] M. A. Pai. Energy function analysis for power system stability. Berlin: Kluwer Academic, 1989. 56 p.

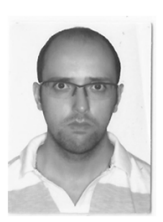

Matheus Henrique Marcolino é graduado em engenharia elétrica pela Universidade Estadual Paulista (UNESP), Campus de Ilha Solteira, São Paulo, Brasil, em 2012. PósGraduado em Engenharia Elétrica em nível de mestrado pela Universidade Estadual Paulista (UNESP), Campus de Ilha Solteira, São Paulo, Brasil, em 2014. Atualmente é pesquisador, em nível de doutorado, do Instituto Tecnológico de Aeronáutica (ITA). Suas áreas de pesquisa são automação, proteção de sistemas elétricos de potência, inteligência artificial e otimização em decisão e controle.

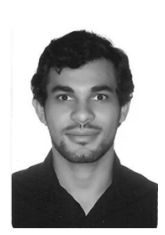

Jônatas Boas Leite é graduado em engenharia elétrica pela Universidade Estadual Paulista (UNESP), Campus de Ilha Solteira, São Paulo, Brasil, em 2010. Atualmente é um estagiário de Pós-doutorado no Programa de Pós-graduação em Engenharia Elétrica da UNESP/Ilha Solteira-SP-Brasil. Suas áreas de pesquisa são o planejamento e controle do sistema de energia elétrica.

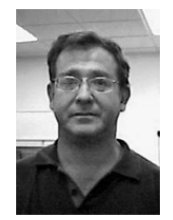

José Roberto Sanches Mantovani é graduado em Engenharia Elétrica pela Universidade Estadual Paulista (UNESP), Campus de Ilha Solteira, São Paulo, Brasil, em 1981. PósGraduado em Engenharia Elétrica em nível de mestrado pela Universidade Estadual de Campinas (UNICAMP), São Paulo, Brasil, 1987. Pós-Graduado, em nível de doutorado, em Engenharia Elétrica pela UNICAMP em 1995. Atualmente é professor titular do Departamento de Engenharia Elétrica (DEE) e membro do Laboratório de Planejamento de Sistemas de Energia Elétrica (LaPSEE) na UNESP. 